TITRE: OBJeCtIVITÉ ET BIENVEILLANCE DU CHERCHEUR À L'ÉGARD DU SUJET DE RECHERCHE Auteur(s): Michel T. GIROUX

Publication: ReCRUtement et CONSENTEMENT À LA RECHERCHE : RÉALITÉS ET DÉFIS ÉTHIQUeS

PAGES: $7-26$

ISBN: 978-2-7622-0357-8

Directeurs: AnA MARIN, BÉATRICE EYSERMANN ET MICHEL T. GIROUX

URI: HTTP://HDL.HANDLE.NET/11143/14104

DOI: HTTPS://DOI.ORG/10.17118/11143/14104 


\section{Objectivité et bienveillance du chercheur à l'égard du sujet de recherche}

Michel T. Giroux, avocat et docteur en philosophie, directeur de l'Institut de consultation et de recherche en éthique et en droit (ICRED), président du Comité d'éthique de la recherche des établissements du Centre de recherche interdisciplinaire en réadaptation du Montréal métropolitain (CRIR), président du Comité d'éthique de la recherche Biobanque Genizon chez Génome Québec, professeur associé au département de médecine sociale et préventive à la Faculté de médecine de l'Université Laval.

Résumé : Celui qui accepte de contribuer à un projet de recherche est fréquemment appelé participant. À partir d'une comparaison entre les mots participant et sujet, voyons lequel désigne le plus adéquatement la personne qui contribue à un projet de recherche. Examinons aussi lequel de ces termes est le plus susceptible d'inspirer au chercheur le respect à l'égard de la personne qui contribue à son projet de recherche. L'enfance et l'inaptitude sont deux conditions inexorables de vulnérabilité. II faut y ajouter le désespoir, qui compromet l'aptitude à décider de façon libre et éclairée. Le phénomène de la méprise thérapeutique résulte d'une incompréhension de la différence entre la finalité de la recherche et la finalité de la clinique. Le chercheur soucieux de recruter des sujets qui se représentent adéquatement son projet de recherche, et qui y consentent véritablement devrait adopter deux attitudes à leur égard : l'objectivité et la bienveillance.

Mots-clés : participant, sujet, méprise thérapeutique, objectivité, bienveillance.

Abstract: The one who agrees to contribute to a research project is frequently called participant. From a comparison between the words participant and subject, let us see which one designates most adequately the person who contributes to a research project. Also let us examine which of these words is the most susceptible of inspiring in the researcher respect towards the person who contributes to his research project. Childhood and inaptitude are two inexorable conditions of vulnerability. It is necessary to add despair, which compromises the capacity to decide in a free and enlightened way. The phenomenon of therapeutic misconception results from the incomprehension of the difference between the purpose of research and the purpose of clinical treatment. The researcher worried of recruiting subjects who understand his research project adequately, and which really agree to it should adopt two attitudes towards them: objectivity and benevolence.

Keywords: participant, subject, therapeutic misconception, objectivity, benevolence. 


\section{Introduction}

La contribution d'un sujet à un projet de recherche peut être motivée par les raisons les plus diverses, qu'elles soient réalistes ou chimériques: contribuer au progrès scientifique, obtenir une attention médicale particulière, profiter d'une meilleure chance de guérison, s'arracher à la détresse, renouveler une espérance amoindrie, rencontrer de nouvelles personnes, se sentir utile à ses concitoyens, etc. De son côté, le chercheur a besoin de personnes qui contribuent à son projet. Ses objectifs peuvent être aussi divers que ceux du sujet. Le microcosme que constitue l'univers de la recherche en santé illustre exactement la nature sociale de l'être humain, en ce que chacun a besoin d'autrui pour se réaliser ${ }^{1}$.

Celui qui accepte de contribuer à un projet de recherche en y investissant sa personne est fréquemment appelé participant. L'appellation de participant peut être appropriée dans le cas d'une personne majeure et apte qui joue un rôle actif dans la réalisation de la recherche. Par contre, convient-elle vraiment à un bébé, à une personne qui a fourni des échantillons ou à une personne devenue inapte? À partir d'une comparaison entre les termes participant et sujet, voyons lequel désigne le plus adéquatement celui qui contribue de sa personne à un projet de recherche. Examinons aussi lequel de ces termes est le plus susceptible d'inspirer au chercheur le respect à l'égard de celui qui contribue de sa personne à son projet de recherche.

Chaque acteur de l'univers de la recherche agit en fonction de sa propre perspective, suivant certaines motivations dont il informe d'autres personnes, mais aussi suivant d'autres motivations qu'il souhaite garder pour lui-même, dans le domaine du non-dit de sa vie personnelle ou professionnelle. Cet état de fait est considéré comme moralement acceptable tant que la relation entre le chercheur et le sujet se conforme aux règles applicables, qu'elles soient légales ou éthiques. En effet, la relation entre le chercheur et le sujet apparaît comme relativement égale ou proportionnée si les mécanismes de protection des sujets sont respectés.

Toutefois, l'égalité formelle relative entre le chercheur et le sujet devient plus difficile à réaliser lorsque le sujet se trouve en situation ou en état de vulnérabilité. L'origine de cette vulnérabilité peut concerner la santé physique ou mentale, la littératie, la relation de couple, l'éducation des enfants, un revers financier, une perte d'emploi, le décès d'une personne chère, l'inadaptation scolaire ou sociale, etc. La condition de vulnérabilité chez une personne peut mettre en péril son intégrité, sa sécurité, son bien-être et l'exercice de ses droits. Nous devons pouvoir compter sur le chercheur et le comité d'éthique de la recherche (CER) pour repérer les personnes en situation de vulnérabilité et leur procurer la protection appropriée.

L'Énoncé de politique des trois Conseils (EPTC2) identifie des personnes qui ont historiquement connu la vulnérabilité : «Les enfants, les personnes âgées, les femmes, les détenus, les personnes souffrant de problèmes de santé mentale et les personnes dont l'aptitude à décider pour elles-mêmes est diminuée» (EPTC2, 2014 : 9). Le dénuement socioéconomique offre aussi un terreau fertile aux abus à l'égard de populations vulnérables. Un exemple classique de ce type d'abus est celui de l'Étude de Tuskegee sur la syphilis².

1. Le philosophe Martin Blais décrit ce besoin que nous avons de nos semblables: «Dire de l'être humain qu'il est social, c'est dire qu'il est fait pour la vie en société en tant qu'il a besoin des autres pour se réaliser, pour s'épanouir ou pour se développer selon toutes ses dimensions». (Blais, 1984, 153).

2. "A classic example of an unjustified offer occurred during the Tuskegee syphilis study. Researchers used various offers to stimulate and sustain the subjects' interest in continued participation ; these offers included free burial assistance and insurance, free transportation to and from the examinations, and a free stop in town on the return trip. Subjects also received free medicines and free hot meals on the days of the examination. The subjects' socioeconomic deprivation made them vulnerable to these overt and unjustified forms of manipulation.» (Beauchamp et Childress, 2013, 139-140). 
Aucune énumération des conditions de vulnérabilité ne peut être exhaustive. Par contre, il est possible d'identifier des conditions inexorables de vulnérabilité, peu importe de qui il s'agit : l'enfance et l'inaptitude. Cette réflexion propose d'ajouter une troisième condition inexorable de vulnérabilité : le désespoir. Notre système juridique vient au secours des enfants et des personnes inaptes en établissant des régimes de protection qui imposent certaines balises aux acteurs chargés de prendre des décisions les concernant. Nos lois prévoient l'intervention de ces régimes de protection dans l'univers de la recherche. Fors les circonstances dans lesquelles leur vie ou leur intégrité est menacée, il n'existe aucune protection juridique pour les personnes acculées au désespoir. Cependant, l'expression de ce constat ne doit pas être interprétée comme un appel pour l'adoption d’une protection légale de toutes les personnes désespérées.

Un événement survenu dans la vie professionnelle du médecin Alexis Carrel montre comment des parents affligés, en désespoir de cause, consentent à une intervention extravagante sur leur bébé. Cette aventure nous conduit à examiner certaines conditions d'existence du consentement libre et éclairé en recherche.

La méprise thérapeutique peut être induite par la manière dont l'équipe de recherche présente son projet, incluant la description du projet contenue dans le formulaire de consentement, ou être le fruit des espoirs du sujet. En fait, le phénomène de la méprise thérapeutique ne provient pas nécessairement du comportement du chercheur. Il est bien souvent le résultat d'une incompréhension de la différence entre la finalité de la recherche et la finalité de la clinique. II arrive aussi que le sujet développe une pensée magique ou exagérément optimiste. Est-il envisageable que le chercheur adopte des attitudes qui réduisent les possibilités de méprise thérapeutique?

Le chercheur soucieux de recruter des sujets qui se représentent adéquatement son projet de recherche et qui y consentent véritablement devrait adopter deux attitudes à leur égard : l'objectivité et la bienveillance. L'ambition de cette réflexion est de montrer que les attitudes d'objectivité et de bienveillance chez le chercheur représentent une solution propre à relever les défis de l'énergie du désespoir et de la méprise thérapeutique.

La teneur de ce propos s'articule autour de quatre événements réels dont les protagonistes essentiels sont des personnes qui se trouvent en situation ou en état de vulnérabilité. Dans la section intitulée «Énergie du désespoir», il est question d'une aventure audacieuse en transfusion sanguine. Dans la section portant sur la méprise thérapeutique, nous rappelons les leçons apprises d'un essai clinique en psychiatrie. Dans la section portant sur l'objectivité, les parents d'un enfant très malade se font offrir la participation à un essai clinique comme représentant la dernière chance pour le jeune patient. Dans la section suivante, une recherche psychosociale auprès de jeunes contrevenants illustre la pertinence de la bienveillance en recherche.

La première partie du développement de ce texte consiste dans un exposé sur la distinction conceptuelle entre la finalité de la recherche et la finalité thérapeutique. La connaissance de cette distinction sera très utile à titre d'entrée en matière et de référence pour la compréhension de la suite du texte, puisque la recherche et la thérapeutique s'y côtoient fréquemment. 


\section{Finalité de la recherche et finalité thérapeutique : distinction conceptuelle}

Du point de vue conceptuel, une intervention à finalité thérapeutique diffère grandement d'une intervention appartenant à la recherche. Le professionnel de la santé qui intervient auprès de son patient dans l'intention de le guérir ou de lui apporter un réconfort agit dans la sphère thérapeutique. Son énergie et sa compétence sont exclusivement tendues vers le bien-être de son patient ou de son client. De son côté, le chercheur qui conçoit ou met en œuvre un projet de recherche a pour premier objectif l'amélioration des connaissances. Toutefois, son attitude tendue vers l'amélioration des connaissances ne signifie pas que le chercheur puisse se montrer indifférent à propos de la condition du sujet de recherche. Au contraire, l'éthique de la recherche contemporaine établit clairement que le respect de l'intégrité et du bien-être du sujet est un impératif incontournable qui a préséance sur les intérêts de la recherche et de la société ${ }^{3}$. C'est pourquoi les bénéfices pour les sujets de recherche doivent être maximisés, et les effets nocifs, réduits au minimum, nous dit l'Organisation des Nations Unies pour l'éducation, la science et la culture (UNESCO)4.

Rappelons toutefois que, dans les faits, certaines initiatives de recherche sont ajoutées aux soins thérapeutiques à titre de voies inédites qui offrent une possibilité de plus longue survie, d'amélioration de la santé, peut-être même de guérison. Des patients acceptent de devenir sujets de recherche parce qu'ils sont porteurs d’un tel espoir. Dans ces cas, la conduite du clinicien embrasse une finalité thérapeutique.

\section{Participant ou sujet de recherche?}

L'EPTC2 pose la question de savoir comment il convient de nommer quelqu'un qui contribue de sa personne au projet de recherche. Participant ou sujet de recherche? Le fait de poser cette question nous rappelle la nécessité de choisir les mots justes ou les plus appropriés dans le contexte de leur emploi. Nous devons les considérer chacun soigneusement dans la visée d'une argumentation limpide qui ne confond pas les termes.

À la question de savoir si une personne doit être appelée participant ou sujet, l'EPTC2 répond qu'il convient de privilégier participant. Voici comment l'EPTC2 définit les participants :

Dans le contexte de la présente politique, les «participants humains» (appelés «participants») sont les personnes dont les données ou les réponses à des interventions, à des stimuli ou à des questions de la part du chercheur ont une incidence sur la question de recherche (EPTC2, 2014 : 14).

L'EPTC2 justifie ainsi sa préférence : participant «reflète mieux l'esprit qui sous-tend les principes directeurs : que les personnes qui choisissent de participer à un projet de recherche y jouent un rôle plus actif que celui qu'évoque le terme "sujet" ». Un autre passage de l'EPTC2 vient préciser, dans le contexte des données et du matériel biologique humain, la notion de participant : «une personne dont les données ou le matériel biologique sont utilisés dans la recherche devient un participant»(EPTC2, 2014 : 195). Ici, la personne concernée est

3. «Researchers should ensure the wishes and interests of the donor, where known, are respected at all times and the welfare of research participants should always take precedence over the interests of science and society.» (MRC, 2014, 5).

4. «Dans l'application et l'avancement des connaissances scientifiques, de la pratique médicale et des technologies qui leur sont associées, les effets bénéfiques directs et indirects pour les patients, les participants à des recherches et les autres individus concernés, devraient être maximisés et tout effet nocif susceptible d'affecter ces individus devrait être réduit au minimum.» (UNESCO, 2005, art. 4). 
un participant même si ce que le chercheur utilise est détaché de sa personne, comme des données ou du matériel biologique.

Comment utilise-t-on le terme participant dans le langage quotidien? Le dictionnaire nous renvoie au verbe participer, pour lequel on trouve les définitions suivantes: «1. Avoir part à quelque chose: Participer aux profits d'une entreprise. - 2. Prendre part à une action, à un sentiment, etc. : Participer aux délibérations. Participer à la joie générale» (Larousse, 1997, 5504). Il s’agit d'avoir part, de prendre part.

Selon la nature de la recherche, l'intensité de la contribution du sujet peut varier considérablement : recevoir un médicament dans le cadre d'un essai clinique, utiliser une nouvelle aide technique en réadaptation, prendre part à une entrevue sur les habitudes de vie ou bien confier un prélèvement sanguin et autoriser l'accès à son dossier médical. Contribuer à un essai clinique engage la personne d'une manière plus active et plus entière que de confier du matériel biologique à une biobanque. Dans le cas de la biobanque, il est fort probable que la personne n'entende jamais parler des utilisations qui seront faites de son matériel biologique. Elle ne tient alors aucun rôle actif. II se peut aussi qu'une biobanque offre à ses sujets de leur transmettre des informations générales sur ses activités. Même dans ce cas, le rôle du sujet demeure essentiellement passif. Quelquefois, des chercheurs souhaitent accéder à des renseignements recueillis par diverses organisations pour d'autres finalités que celles de la recherche. Ces renseignements peuvent concerner la conduite automobile, les accidents du travail, les caractéristiques d'un milieu environnemental, le dossier académique, les victimes d'actes criminels, le recours à l'assistance sociale, les déplacements internationaux, etc. De tels projets sont réalisés dans l'ignorance totale des personnes que ces renseignements concernent. Il est alors difficile de qualifier ces personnes de participantes.

Dans le réseau de la santé et des services sociaux, il arrive fréquemment que la mise en œuvre d'un projet ne nécessite aucun contact avec des personnes, mais suppose la consultation du dossier de l'usager. L'équipe de recherche y a accès avec l'autorisation du directeur des services professionnels de l'établissement ${ }^{5}$. L'usager dont on aura consulté le dossier ne le saura jamais. Ainsi, appeler cette personne un participant semble une exagération de son rôle factuel. Par ailleurs, l'EPTC2 reconnaît clairement cette distinction entre une personne qui participe directement à une recherche et celle qui y participe parce qu'un chercheur utilise ses données ou son matériel biologique (EPTC2, 2014, 6). Rappelons néanmoins que toutes les personnes qui contribuent à la recherche, quelle que soit l'intensité de leur contribution, méritent qu'on respecte leurs droits, comme le droit à la vie privéé.

Voyons maintenant ce qu'est un sujet. La signification d'un mot peut varier suivant le contexte de son emploi, on dit alors que ce mot est équivoque. Toutefois, il est rare que deux définitions d'un mot décrivent des réalités incompatibles, voire contraires. C'est pourtant le cas du mot sujet, nous dit un dictionnaire philosophique :

Selon l'étymologie latine, le sujet réunit deux significations contraires. D'une part est sujet celui qui est assujetti à un pouvoir, pouvoir du père, du roi, du président, de la loi, de l'ordre, des supérieurs hiérarchiques, etc. D'autre part, le sujet est l'être autonome et conscient, souverain dans la mesure où il peut affirmer sa liberté et endosser la responsabilité de ses actes, quoi qu'il sache, ou ignore, des déterminations naturelles, psychologiques, socio-historiques, politiques, qui constituent sa situation singulière, mais ne le conditionnent pourtant pas de manière définitive (Larousse, 2003 : 995).

5. «Le directeur des services professionnels d'un établissement ou, à défaut d'un tel directeur, le directeur général peut autoriser un professionnel à prendre connaissance du dossier d'un usager, à des fins d'étude, d'enseignement ou de recherche.» (LSSS, art 19.1)

6. «Toute personne a droit au respect de sa vie privée.» (Charte, art 5) 
Un dictionnaire historique de la langue française relève les deux mêmes significations. Considérons d'abord la signification de l'être assujetti. Le mot sujet constitue un «emprunt au latin subjectus «soumis», "assujetti», «exposé» et «voisin, proche», participe passé passif de subjicere «placer dessous», «amener à proximité de», «soumettre, subordonner»» (Le Robert, 1998, 3686). Maintenant, soulignons le sens de l'être autonome et conscient: «Le mot entre dans le vocabulaire philosophique au début du XIXe s. d'après l'allemand Subjekt (Kant), de même origine, désignant l'être pensant, considéré comme le siège de la connaissance, par opposition à un objet» (Le Robert, 1998, 3686). Envisagé au sens de l'être soumis, sujet prend l'une des significations du mot objet: «Chose inerte, sans pensée, sans volonté et sans droits, par opposition à l'être humain : On nous traite comme des objets» (Larousse, 1997, 5236). L'usage des mots dans le langage quotidien finit par produire une assimilation entre sujet au sens de l'être soumis et objet à titre de chose inerte. Personne ne soutiendrait qu'en recherche sur la santé humaine, il est admissible de considérer la personne comme si elle était un objet. Il est compréhensible que l'on préfère participant à sujet pour désigner la personne qui accepte de contribuer à la recherche si la signification donnée au mot sujet évoque la soumission ou l'idée d'une personne réduite au statut d'objet.

Les dictionnaires contiennent des définitions de sujet qui renvoient à la recherche scientifique. Un dictionnaire historique montre les différents emplois du terme sujet : un être vivant dont les caractéristiques permettent qu'on réalise sur lui des expériences, un être vivant soumis à l'observation, un cadavre utilisé en anatomie, un être vivant que l'on soigne ${ }^{7}$. Dans ce sens du terme sujet, une personne peut être un sujet d'étude ou d'expérimentation. Un dictionnaire encyclopédique propose de définir sujet comme un «être vivant soumis à l'observation» (Larousse, 1997, 6993). La personne humaine devient sujet de recherche lorsque son être physique, psychique ou social est étudié dans le contexte d'une initiative ayant pour finalité le progrès des connaissances. Suivant cette dernière définition de dictionnaire, le terme sujet s’applique à tous les cas de figure, indépendamment du caractère plus ou moins actif de la contribution de la personne concernée.

L'EPTC2 comporte deux versions officielles : l'une est française, et l'autre, anglaise. Les dernières remarques à propos de l'appellation de participant ou de sujet sont fondées sur les définitions de la langue française. Qu'en est-il de l'anglais? Voyons l'équivalent anglais de chaque mot-clé français employé : participant - participant; sujet - subject; objet - object. Ces dyades ne forment pas de faux amis ${ }^{8}$, puisque les diverses significations de ces termes sont similaires d'une langue à l'autre, comme nous le constatons ci-dessous.

Un participant prend part à quelque chose. En anglais, le participant est «A person who takes part in something» (Oxford, en ligne).

Le terme subject prend les trois significations suivantes, parmi d'autres:

- «Under the authority of» (adjectif);

- «A thinking or feeling entity; the conscious mind; the ego, especially as opposed to anything external to the mind» (nom);

- A person who is the focus of scientific or medical attention or experiment» (nom) (Oxford, en ligne).

\footnotetext{
7. «Parallèlement, sujet désigne un être vivant dont les caractéristiques conviennent pour réaliser certaines expériences (mil. XVIe s.), plus généralement un être vivant soumis à l'observation (v. 1560), spécialement un cadavre utilisé pour l'étude de l'anatomie, et un être vivant que l'on soigne (1778).» (Le Robert, 1998, 3687-3688).

8. Les faux amis anglais-français sont des mots de la langue anglaise qui, par leur forme, en rappellent d'autres dans la langue française, mais dont les significations diffèrent. Par exemple, librairie et library sont de faux amis.
} 
La notion d'objet manifeste, en français, la chose inerte. Selon le contexte, le mot anglais object prend ce sens: «A thing external to the thinking mind or subject». (Oxford, en ligne)

La discussion afin de déterminer s'il faut employer participant ou sujet se pose dans les mêmes termes en anglais et en français. Les éléments conceptuels qui organisent la pensée sont virtuellement identiques.

Appliquant au terme sujet les définitions selon lesquelles il est un être pensant, autonome et conscient, l'utilisation de ce terme ne mutile en rien la dignité de la personne humaine. Le statut de sujet introduit une insistance concernant le devoir de respecter la capacité de décider de la personne, exactement dans l'esprit de l'EPTC2:

Respecter les personnes, c'est reconnaître la valeur intrinsèque de tous les êtres humains; c'est aussi reconnaître que chacun a ainsi droit au respect et à tous les égards qui lui sont dus [...]. L'autonomie comprend la capacité de délibérer au sujet d'une décision et d'agir en conséquence. Respecter l'autonomie, c'est reconnaître la capacité de jugement d'une personne et faire en sorte que la personne soit libre de choisir sans ingérence (EPTC2, 2014, 6-7).

Employé au sens de l'être autonome et conscient, le terme sujet affirme immédiatement la dignité de toute personne et rappelle au milieu de la recherche que chacun mérite d'être respecté en considération de cette dignité inhérente.

\section{Énergie du désespoir}

Un dictionnaire encyclopédique propose cette définition du désespoir : «Perte de toute espérance, abattement total de quelqu'un qui a cessé d'espérer; affliction profonde, détresse, désespérance : Sombrer dans le désespoir» (Larousse, 1997, 2188). Si nous donnons créance à ce dictionnaire, il ne faut pas imaginer que le chagrin du désespoir engendre uniquement un état de torpeur ou d'inaction. L'expression en désespoir de cause signifie «faute de pouvoir recourir à un autre moyen» (Larousse, 1997, 2189). Énergie du désespoir dépeint la «résolution violente qu'inspire une situation désespérée» (Larousse, 1997, 2189). Le désespoir peut faire tendre quelqu'un vers une action énergique et obstinée, même si le moyen retenu n'est pas idéal. C'est ce que montre le récit qui suit.

Alexis Carrel, chirurgien et biologiste français, a vécu de 1873 à 1944. Il fut un pionnier de la chirurgie vasculaire. Il a imaginé de nouvelles techniques chirurgicales et réalisé la mise au point d'aiguilles très délicates pour améliorer la qualité des sutures. Afin de développer son habileté manuelle, il devint apprenti auprès de celle qui avait la réputation d'être la meilleure brodeuse artistique à Lyon, madame Leroudier. Le Nobel de médecine fut décerné au Dr Carrel en 1912. Ses travaux l'ont conduit à Montréal, Chicago, puis au Rockefeller Institute for Medical Research à New York.

En 1908, alors qu'Alexis Carrel se trouve à New York, le Dr Adrian Lambert et ses deux frères chirurgiens lui demandent de sauver un bébé. La femme d’Adrian Lambert vient de mettre au monde une petite fille qui perd du sang par le nez et la bouche depuis plusieurs jours. Le Dr Carrel répond qu'il n'est pas autorisé à pratiquer la chirurgie dans l'État de New York: «l have no license to practice surgery in the state of New York. My patients are only my dogs and cats» (Douglas, 2002, 31). Le Dr Lambert s'engage à prendre l'entière responsabilité légale à ce propos. Il insiste pour que le Dr Carrel le suive immédiatement: «Lambert promised that he would take full legal responsibility, if only Carrel would please come-at once» (Douglas, 2002, 31). Le Dr Carrel pratique une transfusion sanguine directe d'une artère du poignet gauche du père dans la veine poplitée du bébé, derrière le genou, par une suture artère-veine. La petite fille prend aussitôt du mieux. 
Le caractère tout à fait exceptionnel de cet événement saute aux yeux. Nous sommes en 1908 et nous pouvons supposer que les protagonistes se sont comportés au meilleur de leurs capacités à ce moment de leur vie. L'intervention envisagée ne fait pas l'objet d'un projet de recherche qui aurait été soigneusement réfléchi et qui interviendrait comme aboutissement à des travaux antérieurs; nous assistons plutôt à une entreprise humanitaire désespérée.

Pour quelles raisons le Dr Adrian Lambert insiste-t-il tellement afin qu'une intervention aussi hasardeuse soit mise en œuvre? Le bébé est sa fille, et il tient à tout faire pour lui sauver la vie, puisque l'alternative semble être d'attendre son décès. Nous pouvons aussi penser que la perte de cette enfant serait désastreuse pour sa mère. L'état des connaissances ne permet pas d'envisager les séquelles possibles de cette intervention. En outre, la condition future du bébé, sa qualité de vie, ne peut pas entrer dans les considérations entre les bénéfices et les inconvénients.

Du point de vue du Dr Carrel, une intervention, même au bénéfice très incertain, offre une réponse immédiate au désespoir des parents. Si le bébé survit, l'intervention aura épargné aux parents le drame de perdre leur enfant, du moins à court terme. De plus, la situation offre au Dr Carrel la possibilité d'oser une intervention sans précédent, et qui lui permet de satisfaire sa curiosité scientifique. Enfin, cette transfusion sanguine innovatrice pourrait contribuer au progrès des connaissances.

Il ne saurait être question d'évaluer la conduite des quatre médecins concernés selon les normes déontologiques actuelles. Cependant, considérer cet événement sous l'angle de la déontologie applicable maintenant met en relief les normes qui nous semblent les plus fondamentales. Bien que les faits se soient déroulés à New York, nous utiliserons la Loi médicale et le Code de déontologie des médecins du Québec, car nous relevons de la juridiction québécoise. Tenons-nous-en uniquement à quelques-unes de leurs dispositions, puisque la considération des autres règles applicables serait interminable.

Premièrement, son statut professionnel ne permettait pas au Dr Carrel d'exercer la médecine à New York. À cette époque, comme aujourd'hui, le Dr Carrel risquait de se faire reprocher l'exercice illégal de la médecine. Dans le contexte québécois actuel, l'exercice illégal de la médecine fait l'objet de l'article 43 de la Loi médicale. L'engagement du Dr Lambert d'en prendre l'entière responsabilité légale ne se substitue pas au permis d'exercer la médecine. La demande empressée du patient ou celle de son représentant légal dans le but d'obtenir une intervention n'autorise personne à exercer la médecine.

Deuxièmement, le médecin doit «engager pleinement sa responsabilité civile» dans sa pratique professionnelle. L'engagement du père du bébé à prendre la responsabilité légale pour la suite des événements ne permet pas au médecin d'éluder sa responsabilité pour les actes professionnels qu'il posera?.

Troisièmement, l'article 28 du Code de déontologie des médecins requiert que le médecin obtienne un consentement libre et éclairé avant de dispenser un soin :

Le médecin doit, sauf urgence, avant d'entreprendre un examen, une investigation, un traitement ou une recherche, obtenir du patient ou de son représentant légal, un consentement libre et éclairé.

9. «Le médecin doit, dans l'exercice de sa profession, engager pleinement sa responsabilité civile. Il ne peut l'éluder ou tenter de l'éluder, ni requérir d'un patient ou d'une personne une renonciation à ses recours en cas de faute professionnelle de sa part.» (Code de déontologie des médecins, art 11) 
La transfusion sanguine effectuée sur le bébé se présente comme une terra incognita pour les quatre médecins présents. Au-delà de l'hémorragie dont souffrait l'enfant, il devait leur être très difficile de s'avancer pour constater un diagnostic ou envisager un pronostic. La conduite d’Adrian Lambert nous invite à penser que le consentement des parents a été libre, néanmoins faiblement éclairé en raison de la réalité des connaissances médicales à cette époque. Le contexte humain dans lequel se trouvait le couple désespéré d'Adrian Lambert consistait dans un choix entre le décès certain de leur petite fille ou la tentative de la sauver au moyen d'une intervention cliniquement hasardeuse. Ainsi formulée, la question impose de choisir entre la vie et la mort, et elle s'adresse à quelqu'un qui considère n'avoir rien à perdre en osant une intervention. Le problème posé serait passablement différent s'il était possible de tenir compte de la qualité de vie future de l'enfant dans l'éventualité de sa survie.

Si nous considérons cette transfusion sanguine d'après les règles applicables en matière de recherche, une disposition de la déontologie médicale attire forcément notre attention : celle qui requiert l'approbation préalable d'un comité d'éthique de la recherche qui requiert l'approbation préalable d'un CER, étant entendu que celui-ci fonctionne dans le respect des normes en vigueur ${ }^{10}$. Cette exigence du respect des normes en vigueur opère un renvoi à des documents normatifs internationaux, canadiens et québécois ${ }^{11}$. Partant, les comportements exigés des médecins en matière de recherche ne se limitent pas aux obligations que contient le Code de déontologie des médecins. Il va de soi que l'intervention du Dr Carrel n'ait pas fait l'objet d'un examen par un CER. Cette intervention n'a donc pas été évaluée par une instance indépendante en fonction de sa validité scientifique, des bénéfices espérés et des risques anticipés. De nos jours, un médecin dont la conduite s'apparenterait à celle du Dr Carrel durant ces événements serait perçu comme ayant démontré une audace et une insouciance inacceptables.

L'histoire du Dr Carrel, du couple Lambert et de la petite fille met en relief certaines questions que pose l'obtention d'un consentement libre et éclairé lorsque l'interlocuteur du chercheur se trouve dans un état de désespoir. Le patient est-il en mesure de retenir et de comprendre correctement les informations reçues? Perçoit-il la réalité de ce que lui propose le chercheur ou se trouve-t-il dans un état psychique qui exagère le bénéfice possible de la recherche? L'équipe de recherche présente-t-elle le projet comme elle le devrait? Ces questions nous conduisent à discuter de la méprise thérapeutique et de la possibilité que cette méprise invalide le consentement. Quel type de conduite le chercheur peut-il adopter pour réduire l'incidence et l'intensité de la méprise thérapeutique?

\section{Méprise thérapeutique}

Le professeur américain de psychiatrie Paul S. Appelbaum a publié de nombreux articles sur la notion de méprise thérapeutique (therapeutic misconception). Une de ces publications, datant de 1987, décrit comment un patient perçoit sa contribution à un projet de recherche. II s'agit d'un jeune homme qui vient de tenter de se suicider, qui a une longue histoire de relations tumultueuses et qui éprouve des difficultés à maîtriser ses impulsions (impulses). Un psychiatre lui offre de participer à un projet de recherche ayant pour objectif de

10. «Le médecin doit, avant d'entreprendre sa recherche sur des êtres humains, obtenir l'approbation du projet par un comité d'éthique de la recherche qui respecte les normes en vigueur, notamment dans sa composition et dans ses modalités de fonctionnement. Il doit également s'assurer que tous ceux qui collaborent avec lui à la recherche soient informés de ses obligations déontologiques.» (Code de déontologie des médecins, art 31).

11. «L'éthique de la recherche en santé humaine est institutionnalisée, elle comporte des instances présentes notamment dans les universités et dans les établissements de santé et de services sociaux. Au cœur de ces instances, nous trouvons les comités d'éthique de la recherche (CÉR) et les comités scientifiques.» (Giroux, 2010, 114) 
constater si des médicaments peuvent aider au traitement de sa condition. Le patient accepte d'y participer : «Yes, I'm willing to do anything that might help me» (Appelbaum et coll., 1987, 20). Quelques jours plus tard, le psychiatre rencontre à nouveau le jeune homme afin de lui donner plus d'informations sur le projet de recherche. Le psychiatre expose que deux médicaments et un placebo seront utilisés. Les médicaments et le placebo seront attribués au hasard, et même le chercheur ne saura pas ce que chacun reçoit. Le patient écoute les informations et signe le formulaire. Selon toute apparence, le consentement exprimé est libre et éclairé. Cependant, quand on demande au patient pourquoi il a consenti à la recherche, il répond que la médication qu'il recevra sera celle qui peut le mieux améliorer sa condition. Il se figure que le projet de recherche est conçu pour lui venir en aide directement.

Cette description des pensées du jeune homme montre que le phénomène de la méprise thérapeutique ne pourrait pas survenir dans le cadre d'un projet de recherche qui utilise des sujets en bonne santé. La méprise thérapeutique est uniquement possible lorsque la recherche recrute des personnes atteintes d'une pathologie.

La représentation que le jeune homme se fait du projet de recherche ne correspond pas à la réalité. La croyance erronée de la personne affectée par la méprise thérapeutique veut que le projet de recherche ait été conçu pour son bien-être à elle et nie la possibilité que sa contribution à la recherche puisse comporter des inconvénients majeurs ${ }^{12}$. La méprise thérapeutique désigne un état d'esprit présent chez la personne qui accepte de contribuer à un projet de recherche. II s'agit d'une incompréhension de sa contribution à la recherche, puisque la personne voit son bien particulier comme un facteur central dans l'intention du chercheur et qu'elle se trouve en déni face aux inconvénients possibles.

Il importe de rappeler que, dans ce cas, la méprise du patient ne provient pas d'indications floues ou mensongères de la part du psychiatre. C'est le patient lui-même qui teinte sa compréhension des tournures de son espérance. Or, il n'existe aucun moyen qui permette à qui que ce soit d'exercer une emprise sur les représentations qu'entretient cette personne. Toutefois, l'équipe de recherche peut vérifier la compréhension du sujet et fournir des explications de manière à contourner la nervosité ou la faible littératie de cette personne.

Le philosophe Alain nous parle du recrutement militaire à une certaine époque. Beaucoup de jeunes hommes pauvres, mais énergiques semblaient se vendre pour une solde et un uniforme, alors qu'ils s'engageaient d'abord pour des motifs de gloire:

Les recruteurs d'autrefois étaient des marchands d'esclaves. Voltaire nous conte dans Candide l'aventure d'un de ces grands imbéciles qui se vendaient pour un bon déjeuner et une petite masse de monnaie, avec la promesse d'une solde et d'un uniforme. En réalité, ces hommes vigoureux, à l'âge où on s'ennuie, avaient envie de gloire et de voir du pays; sans compter que souvent ils étaient bien pauvres et mangeaient trop pour des pauvres. Je crois pourtant que les motifs de gloire étaient les plus forts, comme ils sont aujourd'hui (Alain, 1970, 1057).

Le jeune homme pauvre ne s'engage pas chez les militaires dans le but de se transformer en chair à canon. Il rêve du mieux : voir du pays et rentrer chez lui glorieux. La même espérance pour le mieux semble entraîner le

12. «Yet when the patient is asked why he agreed to be in the study, he offers some disquieting information. The medication that he will receive, he believes, will be the one most likely to help him. He ruled out the possibility that he might receive a placebo, because that would not be likely to do him much good. In short, this man, now both a patient and a subject, has interpreted, even distorted, the information he received to maintain the view - obviously based on his wishes - that every aspect of the research project to which he had consented was designed to benefit him directly. This belief, which is far from uncommon, we call the 'therapeutic misconception.' To maintain a therapeutic misconception is to deny the possibility that there may be major disadvantages to participating in clinical research that stem from the nature of the research process itself.» (Appelbaum et coll., 1987, 20). 
sujet à croire que la recherche lui offrira, à lui, les meilleurs soins, en dépit des explications très différentes qui lui sont communiquées. Le phénomène de la méprise thérapeutique se produit lorsque la personne concernée ne fait pas la distinction entre la finalité thérapeutique et la finalité de la recherche. Le résultat de cette confusion est de percevoir la contribution à une recherche comme étant de nature thérapeutique.

Une interprétation rigoureuse du critère de la compréhension appropriée a pour effet d'invalider le consentement exprimé, puisque, dans son for intérieur, la personne ne consent pas véritablement à une recherche ${ }^{13}$. Comme l'équipe de recherche n'est pas toujours en mesure de constater cette incompréhension du sujet, nous pouvons supposer que des projets de recherche sont réalisés avec un certain nombre de personnes qui n'ont pas compris ce dont il s'agit. Par ailleurs, quelle devrait être la conduite de l'équipe de recherche qui constate l'absence du caractère éclairé d'un consentement? Serait-il paternaliste d'annoncer au sujet qu'il ne participera pas à la recherche en raison de l'absence du caractère éclairé de son consentement?

L'EPTC2 propose une approche susceptible de limiter le nombre de circonstances dans lesquelles survient la méprise thérapeutique. Il s'agit par exemple de faire en sorte que l'équipe soignante œuvrant auprès de la personne n'intervienne pas ou intervienne aussi peu que possible dans la conduite de la recherche. Lorsque le clinicien traitant participe à la recherche, les responsables de ce projet doivent indiquer quelles mesures seront mises en œuvre pour réduire la possibilité de méprise thérapeutique ${ }^{14}$.

\section{Objectivité et bienveillance chez le chercheur}

Deux attitudes du chercheur et de son équipe contribuent à la possibilité, pour le sujet potentiel, de comprendre adéquatement la recherche et d'y participer en exprimant une volonté libre et éclairée : l'objectivité et la bienveillance. Au sens de son emploi dans ce texte, une attitude consiste dans une «manière d'être qui manifeste certains sentiments» (Larousse, 1997, 539). L'attitude de l'objectivité manifeste l'objectivité, l'attitude de la bienveillance manifeste la bienveillance.

\section{Objectivité}

Voyons d'abord l'objectivité. D'entrée de jeu, précisons que l'objectivité dont il est ici question ne constitue pas une qualité de la connaissance, mais une attitude du chercheur à l'égard de celui qui contribue de sa personne à la recherche, le sujet.

La clarté de ce propos requiert une définition précisant la signification du mot objectivité dans le présent contexte. La notion d'objectivité prend diverses significations, relativement similaires. Une première signification se rapporte à l'indispensable collaboration entre les êtres humains. La simple satisfaction de nos besoins

13. "The 'therapeutic misconception' is a widely discussed problem of informed consent that must be addressed in research, where subjects may fail to distinguish between clinical care and research and may fail to understand the purpose and aim of research, thereby misconceiving their participation as therapeutic in nature. In a stringent interpretation of the standard of adequate understanding, the therapeutic misconception invalidates a subject's consent because he or she is not truly consenting to participation in research.» (Beauchamps et Childress, 2013, 133).

14. «En règle générale, on peut réduire la possibilité que les participants ne se méprennent sur l'aspect thérapeutique d'un essai et éviter ainsi de créer un malentendu ou de fausses attentes, si les cliniciens qui dispensent des soins de santé au patient interviennent aussi peu que possible dans le recrutement et le processus de consentement. Idéalement, les fonctions de traitement et de recherche seront assumées par des personnes différentes, mais dans certains cas, il sera dans l'intérêt des participants d'impliquer leur clinicien traitant au processus de recrutement et de consentement. Dans ces cas, le dossier du projet de recherche proposé doit indiquer les mesures supplémentaires qui seront prises pour réduire la possibilité de méprise thérapeutique.» (EPTC2, 2014, 183). 
vitaux nous impose de nous adapter à la réalité, de communiquer avec d'autres personnes et de coopérer avec elles sur le plan pratique, nous dit le philosophe José Antonio Marina. La mise en œuvre de toutes ces composantes requiert la configuration, dans la conscience du sujet, d'un espace objectif, commun, interpersonnel et durable. Le dialogue, par exemple, est possible seulement si on peut sortir, au moins de façon partielle, de son monde privé pour accéder à l'objectivité, un espace interpersonnel que personne ne possède, et accessible à tous ${ }^{15}$. Prise dans ce sens, l'objectivité résulte de la sortie de soi-même. II s'agit d'adopter une posture psychique qui renonce au confort de la subjectivité et qui s'efforce d'entrer en contact avec l'autre au moyen d'un langage commun.

Un dictionnaire terminologique propose une définition de l'objectivité qui la considère comme une préoccupation : «Souci constant de faire abstraction des mobiles et des opinions personnelles dans le jugement» (OLF, en ligne). Cette définition met en évidence l'intention de la personne pour laquelle il importe de faire abstraction de ses opinions personnelles dans le jugement qu'elle pose. Il s'agit d'un «souci», d'une préoccupation, plutôt que d'un accomplissement. L'objectivité ainsi définie est l'affaire de toutes les activités professionnelles : de la pratique médicale à la sociologie, de la pratique du droit à l'ingénierie. Définie comme une préoccupation, l'objectivité ne présente point le caractère de la certitude mathématique ni son auréole d'inaccessibilité pour les chercheurs des autres sciences. À titre de préoccupation, l'objectivité est universellement réalisable, au prix d'une attention soutenue.

Un autre dictionnaire formule une définition qui exprime un niveau d'exigence plus élevé que celui de la préoccupation, en ce que la personne concernée porte effectivement un jugement sans qu'interviennent ses opinions ou ses préférences personnelles : «Qualité de quelqu'un, d’un esprit, d'un groupe qui porte un jugement sans faire intervenir des préférences personnelles: Diriger avec objectivité des débats.» (Larousse, 1997, 5236). Nous trouvons des illustrations de cette objectivité dans la décision juste d'un tribunal et dans l'impartialité du président d'une assemblée.

Enfin, l'objectivité peut nommer une caractéristique désirable de l'activité intellectuelle. Il s'agit alors de la «Qualité de ce qui est conforme à la réalité, d'un jugement qui décrit les faits avec exactitude : L'objectivité d'un récit» (Larousse, 1997, 5236). Parvenir à une description exacte du réel constitue un défi considérable pour tous les domaines de connaissance. De l'astrophysique à l'anthropologie, les chercheurs observent et tentent d'expliquer divers phénomènes. Les abonnés à la littérature scientifique constatent rapidement que telle description d’un phénomène céleste peut être améliorée, que tel médicament provoque des réactions positives imprévues ou que telle intervention de la psychologie se révélerait peut-être plus efficace si elle était modifiée en fonction d'une approche innovatrice. La conquête de nouveaux savoirs se révèle difficile dans un contexte où les chercheurs conduisent leurs travaux en tâchant de décrire les faits avec exactitude. Néanmoins, l'évolution du savoir serait un projet absurde si chacun y allait de sa subjectivité particulière.

Maintenant, illustrons l'objectivité comme attitude du chercheur à l'égard du sujet.

Afin de complexifier un petit peu notre enjeu, situons-le en pédiatrie, à propos d'un enfant de deux ou trois ans. La décision de participer ou non à une recherche appartient aux parents, bien que le sujet éventuel ne soit aucun d'eux, mais leur enfant. Les parents d'un enfant atteint d'une maladie grave à qui on offre de participer à un essai clinique peuvent être vivement tentés d'y consentir si cet essai clinique leur est présenté comme l'inter-

15. «Las necesidades vitales imponen una adecuación a la realidad, una comunicación con otros seres y una cooperación con ellos en el plano práctico. Todas estas cosas exigen la configuración en la conciencia del sujeto de un espacio objetivo, común, interpersonal y firme. El diálogo, por ejemplo, sólo es posible cuando puede salirse, aunque sea fragmentariamente, del mundo privado para acceder a la objetividad, una tierra de nadie utilizable por todos.» (Marina, 2004, 52). 
vention de la dernière chance. Ajoutons à cette justification le postulat selon lequel des parents qui veulent le mieux pour leur enfant devraient évidemment y consentir. Les subtilités des explications données aux parents exercent une influence déterminante sur leur décision, certes, de même que sur la qualité de leur décision. Dans les pires cas, le chercheur qui présente son projet de recherche de manière à obtenir un consentement subordonnera, tôt ou tard, son objectivité à son intérêt. Face à des parents très inquiets, voire désespérés, l'objectivité du chercheur qui leur transmet les renseignements leur permet de consentir à une intervention ou de la refuser en sachant de quoi il retourne.

Comme de raison, les parents auront tendance à rechercher l'opinion du médecin traitant, car celui-ci connaît leur enfant et détient des connaissances scientifiques de même qu'une expérience clinique. De plus, même si son activité le rapproche des parents, ce médecin n’est pas engagé personnellement dans la situation familiale. Il lui est plus facile qu'à un proche d'exprimer un avis à la fois détaché et conforme à ce que la réalité scientifique rend possible. Le rôle du médecin traitant est d'utiliser sa connaissance au service de son petit patient et des parents : «Dans une situation aussi difficile, le médecin spécialiste jouit d'un grand avantage sur les parents : lui, il sait. Certes, il ne sait pas tout, mais il détient des connaissances» (Dorval et Hébért, 2005, 59) sur les jeunes patients en général et sur les particularités de l'enfant dont il s'agit. Les parents lui font spontanément confiance.

Le souci constant de faire abstraction de ses opinions et de ses mobiles personnels requiert du chercheur qu'il s'efforce de ne pas se laisser influencer par son a priori intellectuel ou son intérêt. Par exemple, il peut être tentant pour un chercheur dans le champ biomédical d'appliquer une interprétation créative des critères d'inclusion à un projet de recherche. C'est la raison pour laquelle l'Association médicale canadienne estime que la rémunération en conséquence du recrutement n'est pas éthique. Il en va de même pour l'établissement de quotas en rapport avec la rémunération ${ }^{16}$. Dans le même esprit, l'EPTC2 demande au CER d'examiner les modalités de paiement afin d'éviter un recrutement inapproprié ${ }^{17}$. Il reste que les instruments les plus efficaces pour prévenir de tels abus demeurent l'objectivité et l'honnêteté du chercheur qui s'emploie à mettre de côté son intérêt pécuniaire.

Maintenant, supposons qu'un chercheur d'une discipline des sciences humaines ait développé la conviction que, dans leur propre intérêt, les itinérants devraient faire l'objet d'interventions psychosociales beaucoup plus autoritaires et contraignantes. Pour convaincre son milieu professionnel de la justesse de sa conviction, le chercheur élabore un projet de recherche dont les instruments de collecte de données et les outils d'analyse comportent des biais destinés à confirmer son approche de l'itinérance. Dans sa description du projet aux sujets potentiels, ce chercheur procédera sans objectivité et, de ce fait, il obtiendra des consentements non éclairés.

La nécessité morale de l'objectivité au sens d'une préoccupation face aux préférences et aux opinions personnelles permet au chercheur de repousser la tentation de l'avidité et l'appel de la vanité. Une carrière scienti-

16. «Are there financial incentives for researchers to recruit participants? Finders' fees and referral fees should not be accepted; they are unethical. Are there rewards for meeting quotas? This may unduly influence recruitment.» (CMA, 2008, 17).

17. «Le CER examinera les budgets pour vérifier qu'aucun paiement inapproprié ou que d'autres dépenses inexpliquées ne permettent de soupçonner l'existence de conflits d'intérêts. En outre, il étudiera les dispositions de paiement pour veiller à ce qu'elles n'entraînent aucun incitatif de recrutement rapide, inacceptable sur le plan éthique, au détriment d'une étude minutieuse des caractéristiques recherchées chez les participants éventuels. Dans le cas de paiements déraisonnables ou d'incitations indues, le chercheur, et parfois son établissement d'attache, pourrait se trouver dans une situation de conflit entre la recherche d'une rémunération financière maximale, d'une part, et la protection des participants ainsi que le respect des objectifs et exigences scientifiques du projet de recherche, d'autre part.» (EPTC2, 2014, 105). 
fique peut être aussi politique, compétitive et féroce que toute autre carrière. Elle est parsemée d'invitations à utiliser des expédients faciles ${ }^{18}$. Nous rejoignons ici notre point de départ en compagnie du philosophe Marina: L'accès à l'objectivité requiert l'adoption de la posture psychique de celui qui sort de lui-même pour accéder au monde extérieur, à des relations interpersonnelles et à des pensées autres qu'égocentriques.

\section{Bienveillance}

Passons maintenant à la bienveillance. Quiconque s'intéresse à la bioéthique rencontre naturellement quatre principes largement connus : respect de l'autonomie, bienfaisance, non-malfaisance et justice. Il est régulièrement question du principe de bienfaisance au cours de lectures ou lors de conversations à propos de la relation thérapeutique. Quant à elle, la bienfaisance doit être distinguée de la bienveillance, car il existe une différence appréciable entre ces deux termes, que l'on risque de confondre par mégarde. L'étymologie nous renseigne adéquatement sur la distinction à établir entre eux. La bienfaisance (bien et faire) consiste dans l'action de faire du bien, alors que la bienveillance (bien et vouloir) (Larousse, 2003, 392-393) est l'attitude de la personne qui montre «de bonnes dispositions à l'égard de quelqu'un» (Larousse, 1997, 826). Comme il a déjà été expliqué, à la différence du contexte thérapeutique dont la visée est de procurer un bien au patient, le contexte de la recherche a pour objectif l'amélioration des connaissances. Il se peut que sa contribution à une recherche améliore la condition d'une personne, mais ce résultat n'est pas le premier objectif de la démarche du chercheur. Conséquemment, on ne peut exiger du chercheur qu'il contribue au bien-être des sujets, qu'il soit bienfaisant, dans la réalisation de son projet:

Il arrive souvent qu'un projet de recherche n'offre guère ou n'offre pas de bénéfices directs aux sujets. En fait, les principaux bénéfices qui découlent de la plupart des projets de recherche concernent la société et l'avancement des connaissances (EPTC2, 2014, 22)

Jusqu'où la bienveillance du chercheur est-elle susceptible de se manifester? Se trouve-t-elle adéquatement inspirée lorsqu'elle tient compte de la perspective ou de la personnalité du sujet? La personnalisation d'une activité consiste dans l'«adaptation d'un produit, d'un service, d'un logement, etc., à la personnalité de celui à qui il est destiné» (Larousse, 1997, 5623). Est-il vraisemblable que, dans l'élaboration et la réalisation d'un projet de recherche, le chercheur tienne compte de la personnalité du sujet, de son point de vue? Le défi de la bienveillance en recherche apparaît au premier chapitre de l'EPTC2, lorsque ce document normatif affirme que les chercheurs et les CER ont l'obligation de tenir compte de la perspective du participant:

Dans l'élaboration et la réalisation d'une recherche ou lors de son évaluation éthique, les chercheurs et les CER ont l'obligation de tenir compte de la perspective du participant. En conséquence, il pourrait être nécessaire de se pencher sur divers contextes (par exemple social, économique, culturel) qui façonnent la vie du participant pour évaluer convenablement les implications de la recherche au regard des principes directeurs (EPTC2, 2014, 11).

Ce défi de la bienveillance apparaît une seconde fois dans l'EPTC2, concernant les avantages, les risques ou les inconvénients que comporte un projet. L'EPTC2 recommande clairement aux chercheurs et aux CER d'adopter l'attitude appelée ici bienveillance:

18. «Science, I had come to learn, is as political, competitive, and fierce a career as you can find, full of the temptation to find easy paths.» (Kalanithi, 2016, 100). 
Les participants peuvent percevoir les préjudices d'une façon différente des chercheurs. Ils peuvent aussi réagir de façons différentes à la recherche. Les chercheurs et les CER devraient autant que possible tenter d'évaluer les préjudices du point de vue des participants (EPTC2, 2014, 22).

Le premier devoir de la bienveillance est certainement de protéger les sujets contre tout risque inutile ou évitable. Comme pour l'accès à l'objectivité, l'exercice de la bienveillance requiert l'adoption de la posture psychique de celui qui sort de lui-même. Ici, le chercheur sort de lui-même en s'enquérant du point de vue d'une autre personne, le sujet.

Il peut être aisé de saisir le point de vue du sujet, mais plus complexe d'en tenir compte avec justesse, même dans une intention bienveillante. Une illustration qui comporte sa part d'humour nous le montre bien. Un psychologue souhaite réaliser un projet de recherche auprès de jeunes contrevenants qui se trouvent en centre jeunesse. Sa recherche comporte un entretien avec chaque sujet. Pour inciter les jeunes à participer et en guise de reconnaissance pour leur contribution, le chercheur leur offre un sac de chips, une boisson gazeuse et une barre de chocolat. Il faut savoir que l'offre alimentaire du centre jeunesse ne comporte ni croustilles, ni boissons gazeuses, ni barres de chocolat. Il est manifeste que ces régals exerceront une puissante séduction chez les jeunes.

Durant la rencontre du CER qui évalue ce projet, les membres expriment divers points de vue. Certains rappellent que les croustilles, les boissons gazeuses et les barres de chocolat présentent des inconvénients démontrés par rapport à la santé. Des collègues répliquent en alléguant ce qui leur semble de sens commun : ce n'est pas une consommation aussi exceptionnelle de malbouffe ${ }^{19}$ qui risque de nuire à leur santé. Puis, l'argument de l'exemplarité est invoqué. Il ne faudrait pas qu'un chercheur se comporte de manière à confirmer que la malbouffe constitue un modèle dans l'alimentation. On réplique à cet argument que, pour la plupart, les jeunes qui participeront en ont vu d'autres et ne sont pas si crédules. Enfin, un membre du CER soutient qu'offrir ces aliments, c'est miser sur une certaine faiblesse de caractère chez les jeunes approchés, abuser d'eux par un appel à la gourmandise, plutôt que de stimuler leur altruisme et leur désir de vivre en santé. Il ajoute qu'il s'agit là d'une incitation trop séduisante pour ces jeunes et conclut son laïus en citant un passage de l'EPTC2 selon lequel on doit éviter «que les participants éventuels perçoivent les incitations comme une façon de gagner des faveurs ou d'améliorer leur situation» (EPTC2, 2014, 29). Un autre membre du CER souligne que la règle à respecter est de se limiter au versement d'une indemnité compensatoire, comme le prévoit le code civil du Québec ${ }^{20}$.

Les membres des CER sont susceptibles d'adopter des postures de départ infiniment variées, censément en fonction de leurs sensibilités, de leurs valeurs et de leurs expériences. Certains sont portés à faire volontiers confiance aux équipes de recherche, alors que d'autres ne dissimulent point leur forte inclination pour la pratique du materno-paternalisme à l'égard des sujets.

19. «Nourriture malsaine en raison de sa faible valeur nutritive et de sa teneur élevée en sucres ou en gras.» (OLF, en ligne).

20. La règle de l'indemnité compensatoire apparaît à l'article 25 du Code civil du Québec:

«L'aliénation que fait une personne d'une partie ou de produits de son corps doit être gratuite; elle ne peut être répétée si elle présente un risque pour la santé.

«La participation d'une personne à une recherche susceptible de porter atteinte à son intégrité ne peut donner lieu à aucune contrepartie financière hormis le versement d'une indemnité en compensation des pertes et des contraintes subies.» 
Dans le cas de notre illustration en centre jeunesse, un chercheur ou un membre de CER peut-il légitimement présumer de la perception des sujets potentiels? Comment un chercheur ou un membre de CER animé par la bienveillance doit-il aborder une telle question? Pour celui qui adopte de bonnes dispositions à l'égard des sujets potentiels, une démarche appropriée pourrait être de poser directement la question de son point de vue au sujet ou à des personnes appartenant au même groupe que le sujet. Passant ainsi d'un point de vue présumé à la connaissance d'un point de vue exprimé, la personne animée par la bienveillance accomplirait un progrès important pour atteindre l'objectif d'une application circonstanciée des normes congruentes.

Une illustration juste de l'intention derrière cette bienveillance concerne le don d'échantillons biologiques. Le Medical Research Council (conseil) affirme que la recherche portant sur de tels échantillons devrait être conduite avec respect et transparence à l'égard des donneurs. Le conseil propose qu'avant d'approcher des donneurs potentiels les chercheurs devraient être conscients qu'il puisse y avoir des différences individuelles, culturelles ou religieuses concernant la signification et l'importance du corps humain ou de certaines de ses parties $^{21}$.

\section{Conclusion}

L'EPTC2 pose la question de savoir comment il convient de nommer quelqu'un qui contribue de sa personne à un projet de recherche. Participant ou sujet de recherche? L'EPTC2 retient participant, puisque les personnes qui choisissent de contribuer à un projet de recherche y jouent un rôle plus actif que celui qu'évoque le terme sujet.

Selon la nature de la recherche, l'intensité de la contribution du sujet peut varier considérablement. Contribuer à un essai clinique engage la personne d'une manière plus active et plus entière que confier du matériel biologique à une biobanque. Fréquemment, des chercheurs souhaitent accéder à des renseignements recueillis par diverses organisations pour d'autres finalités que la recherche. De tels projets sont réalisés dans l'ignorance totale des personnes que ces renseignements concernent. Il est alors difficile de qualifier ces sujets de participants.

Dans tout projet, celui qui contribue de sa personne à une recherche doit être traité comme un sujet au sens de l'être autonome et conscient, porteur d'une dignité inhérente. Il faut préférer sujet à participant pour trois raisons:

21. "Samples of human biological material should be treated as donations and research involving these samples should be conducted with respect and transparency.The human body and its parts should be treated with respect, and before approaching potential donors, researchers should be aware there may be individual, cultural or religious differences in the meaning and significance attached to the body or specific parts of it. Researchers should aim to achieve an environment of trust and respect with participants, recognising the altruism of providing samples for use. This environment can be fostered by maintaining a high level of transparency in every area of research practice e.g. from consent to the potential future use of samples, and dissemination of research results.» (MRC, 2014, 6) 
- l'observateur ne peut que constater l'infinie diversité des initiatives de recherche, alors que la contribution attendue de la personne varie considérablement. Parfois, la personne concernée n'entendra jamais parler du projet, parfois sa contribution au projet présentera des risques significatifs qu'elle aura préalablement acceptés. Le terme participant est peu approprié pour identifier des contributions tellement différentes,

- la définition philosophique de sujet au sens de l'être autonome et conscient rappelle au chercheur la dignité intrinsèque de celui qui contribue directement de sa personne ou par l'entremise de renseignements le concernant. Le vocable participant ne comporte pas la richesse de signification du terme sujet,

- entre les termes participant et sujet, le terme sujet - au sens philosophique de l'être autonome et conscient - est le plus susceptible d'inspirer au chercheur le respect à l'égard de celui qui contribue de sa personne à son projet de recherche.

Une règle générale issue des normes éthiques et juridiques requiert que la contribution d'un sujet à un projet de recherche soit précédée par l'expression de son consentement libre et éclairé. Cette règle s'impose, sauf pour les exceptions que la loi et les normes éthiques identifient. Suivant l'expérience commune, nous pouvons supposer que la plupart des sujets potentiels sont en mesure de comprendre les explications du chercheur et de délibérer de façon libre pour décider d'accepter ou de refuser la contribution qui leur est proposée. Néanmoins, l'histoire de la recherche sur des sujets humains permet de tirer une leçon : certaines personnes se trouvent dans une position de vulnérabilité inexorable. Parmi elles se trouvent les enfants et les personnes inaptes. À ces deux groupes, il semble opportun d'ajouter la personne en proie au désespoir, celle qui estime ne plus avoir rien à perdre, qu'il s'agisse d'elle-même ou de quelqu'un qu'elle aime.

L'aventure new-yorkaise du Dr Carrel survenue en 1908 illustre parfaitement un consentement qu'expriment des parents habités par le désespoir. L'intervention tentée ne se situait pas dans l'environnement scientifique et éthique qui encadre aujourd'hui la recherche. Toutefois, cet événement révèle comment des personnes plus informées que la moyenne des gens osent envisager un recours dont la pulsion est l'énergie du désespoir. Si le bébé survit, l'intervention aura prolongé une existence humaine et épargné aux parents le drame de perdre leur enfant, du moins à court terme.

Partant de cette conscience du désespoir, observons comment d'autres contextes poignants nous renseignent sur les motivations des sujets de recherche : la littérature médicale fournit des témoignages à ce sujet. Le professeur américain de psychiatrie Paul S. Appelbaum a publié de nombreux articles sur la notion de méprise thérapeutique. Une de ces publications, datant de 1987, décrit comment un patient conçoit sa contribution à la recherche en psychiatrie. Selon toute apparence, le consentement exprimé est libre et éclairé. Cependant, quand on demande au patient pourquoi il a consenti à la recherche, il répond que la médication qu'il recevra sera celle qui peut le mieux améliorer sa condition, manifestant ainsi une méprise thérapeutique.

Deux attitudes du chercheur et de son équipe peuvent contribuer à la possibilité, pour le sujet potentiel, de participer à la recherche en exprimant une volonté libre et éclairée : l’objectivité et la bienveillance.

Le chercheur soucieux d'objectivité s'adresse au sujet potentiel en recourant à la persuasion plutôt qu'à la séduction. La persuasion sollicite l'intelligence d'une personne : elle utilise une argumentation rationnelle dans l'intention de la convaincre. La séduction lorgne du côté des émotions, de la vie psychique. Dans sa personnification la plus terrible, le séducteur n’hésite nullement à «exercer sur quelqu'un tous les moyens de plaire, en particulier pour l'abuser, faire illusion, le faire agir dans un sens bien précis» (Larousse, 1997, 6680). Au fil de la vie quotidienne, chacun constate que la beauté et l'argent exercent un pouvoir de séduction. Dans l'univers de 
la recherche, il est possible pour le chercheur d'employer un discours séducteur, un boniment, qui enchante par les nuances agréables et prometteuses, mais qui néglige de présenter avec objectivité ou franchise les risques et les inconvénients d'un projet.

La bienveillance est l'attitude de la personne qui montre de bonnes dispositions à l'égard de quelqu'un. Est-il possible, dans la réalisation d'un projet, de tenir compte de la personnalité du sujet, de son point de vue, concernant les avantages, les risques ou les inconvénients que comporte la recherche? Une première démarche appropriée pourrait être de poser directement la question de son point de vue au sujet.

L'objectivité et la bienveillance fournissent une inspiration éthique au chercheur, elles bonifient sa pratique professionnelle, et leur exercice constitue une sauvegarde pour les sujets de recherche. 


\section{Bibliographie}

Alain. (1970). Propos, Tome 2, Pléiade, no 619.

Appelbaum, P.S., Roth, L.H., Lidz, C.W., Benson, P. et Winslade, W. False Hopes. (1987). Best Data : Consent to Research and the Therapeutic Misconception. Hastings Center Report, 1987, 17(2) : 20-4.

Beauchamp T.L. et Childress J.F. (2103). Principles of Biomedical Ethics, $7^{e}$ éd., Oxford University Press.

Blais, M. (1984). Une morale de la responsabilité, Fides.

Canadian Medical association (CMA). (2008). Research Guidelines : a web-based decision guide for physicians. Repéré à https://www.cma.ca/Assets/assets-library/document/en/Final_Toolkit_Research-e.pdf

Charte des droits et libertés de la personne (Charte), RLRQ, c. C -12.

Code civil du Québec (CCQ), RLRQ, c. CCQ-1991.

Code de déontologie des médecins, RLRQ, c. M -9, r. 17.

Le Robert. (1998). Dictionnaire historique de la langue française, Paris.

Dorval J. et Hébert B. (2005). Quelques balises éthiques pour composer avec le sort incertain des extrêmes prématurés. Dans Michel T. Giroux et al., L'extrême prématurité. Les enjeux parentaux, éthiques et légaux, (59-68). PUQ.

Conseil de recherches en sciences humaines du Canada, Conseil de recherches en sciences naturelles et en génie du Canada, Instituts de recherche en santé du Canada (décembre 2014). Énoncé de politique des trois Conseils : Éthique de la recherche avec des êtres humains. (cité dans le texte comme EPTC2) http://www.ger.ethique.gc.ca/pdf/fra/eptc2-2014/EPTC_2_FINALE_Web.pdf.

Giroux, M.T. (2010). Cultiver des attentes pragmatiques à l'égard des comités d'éthique». Dans Trudel P. et S.Jean, M. (ed.) La malréglementation. Une éthique de la recherche est-elle possible et à quelles conditions? (113-128) PUM.

Larousse. (2003). Grand dictionnaire de la philosophie. Paris

Larousse. (1997). Grand Usuel Larousse, dictionnaire encyclopédique, Paris.

Kalanithi, P. (2016). When Breath Becomes Air, New York: Random House, New Yok.

Loi médicale, RLRQ, c. M-9.

Loi sur les servies des santé et les services sociaux (LSSSS), RLRQ, c. S-4.2.

Marina, J.A. (2004). La inteligencia fracasada. Teoria y práctica de la estupidez. Editorial Anagrama.

Medical Research Council (MRC). (2014, novembre). Human Tissue and Biological Samples for Use in Research: Operational and Ethical Guidelines, MRC Ethics Series.

Office de la langue française (OLF), Grand dictionnaire terminologique.

Organisation des Nations Unies pour l'éducation, la science et la culture (UNESCO). (2005). Déclaration universelle sur la bioéthique et les droits de l'homme.

Oxford. English Oxford Living Dictionaries. Repéré à https://en.oxforddictionaries.com/

Starr, D. (2002). Blood; An Epic History of Medicine and Commerce, Harper. 